\section{Intramuscular diclofenac for the prevention of post-ERCP pancreatitis: A randomized trial}

\author{
Se Woo Park, Moon Jae Chung, Tak Geun Oh, \\ Jeong Youp Park, Seungmin Bang, \\ Seung Woo Park, Si Young Song
}

Endoscopy 2015;47:33-39

Post endoscopic retrograde cholangiopancreatography (ERCP) pancreatitis (PEP) is one of the potentially serious complications of ERCP with its frequency approaching $30 \%$ in patients with high risk conditions like sphincter of Oddi dysfunction. ${ }^{[1,2]}$ A number of strategies like identifying patients having high risk for PEP, appropriate selection of patients for ERCP using noninvasive or minimally invasive methods such as magnetic resonance cholangiopancreatography or endoscopic ultrasonography (EUS), various pharmacological agents and use of pancreatic stents have been used to decrease the frequency as well as severity of PEP. ${ }^{[3-5]}$ A number of pharmacological agents have been evaluated for prevention of PEP with rectal administration of non-steroidal anti-inflammatory agents (NSAIDs) shown to have promise in decreasing the frequency as well as the severity of PEP. ${ }^{[3-5]}$ The NSAIDs are presumed to be beneficial in prevention of PEP by inhibiting phospholipase A2, cyclooxygenase, and neutrophil-endothelial interactions thereby disrupting the inflammatory cascade of acute pancreatitis. ${ }^{[3-5]}$

A number of randomized studies as well as meta analysis have demonstrated the safety as well efficacy of NSAID's in prevention of PEP. ${ }^{[6-8]}$ However, interestingly majority of these trials have used rectal route of NSAID's and it is unclear whether parenteral route of administration is as effective as rectal NSAID's. A prospective, randomized, placebo-controlled study of 80 patients compared receive parenteral diclofenac at a loading dose of $75 \mathrm{mg}$ followed by the infusion of $5-10 \mathrm{~mL} / \mathrm{kg}$ per hour isotonic saline over $4 \mathrm{~h}$ after the procedure, with infusion of $500 \mathrm{~mL}$ isotonic saline as placebo and found that the overall incidence of pancreatitis was similar between the two groups but in the patients without sphincter of Oddi dysfunction (SOD) PEP was significantly lower in the diclofenac group than in the control group $(P=0.047) .{ }^{[9]}$

The authors of the current study conducted a randomized, single centre, double-blinded study to assess the efficacy of prophylactic intramuscular diclofenac in reducing the incidence and severity of PEP in 383 patients undergoing ERCP. Following enrollment in the study, the patients were randomized to receive either a 90- $\mathrm{mg}$ intramuscular diclofenac bolus dose (given as a 2-mL ampule) immediately following ERCP (diclofenac group) or a 2-mL saline bolus only (placebo group). The serum amylase levels were measured at baseline, and at 4 hours and 18-24 hours after the procedure.

The primary outcome of the study was the incidence of PEP, defined as serum amylase level at least three times the upper limit of the normal range along with newly developed or worsened pancreatic-type abdominal pain and tenderness with nausea and/or vomiting for more than 24 hours after ERCP.

Out of randomized 383 patients, 37 patients were excluded and finally 343 patients were eligible for analysis (173 patients in diclofenac group and 170 patients in placebo group). Majority of the patients included in the study were average risk patients for PEP having choledocholithiasis or malignant obstruction with only $25 \%$ patients having high-risk features such as precut sphincterotomy, pancreatic sphincterotomy, or papillectomy and none of the patients having SOD. The two groups had similar demographic as well as procedural profile including age, sex, body mass index, laboratory tests, presence of periampullary diverticula, total procedure time, selective deep cannulation time, frequency of endoscopic sphincterotomy, insertion of biliary and plastic stents as well as indications for ERCP.

The frequency of PEP was $12.2 \%$ with it being mild in 37 patients $(10.8 \%)$, moderate in 3 patients $(0.9 \%)$, and severe in 2 patients $(0.6 \%)$ with all 42 patients recovering with conservative treatment. There was no significant difference in the frequency of PEP between the two groups (diclofenac group vs. placebo group: 22/173 [12.7\%] vs. $20 / 170[11.8 \%] ; P=0.87)$. Two cases of severe pancreatitis were observed in the diclofenac group and three cases of moderate pancreatitis were seen with one in the diclofenac group and two in the placebo group. The authors also analyzed potential risk factors for PEP and on multivariate logistic regression analysis multivariate logistic regression analysis identified male sex (for female sex compared with male sex: OR $0.350,95 \%$ CI $0.154-0.794$; $P=0.012$ ) and EPBD (OR 3.443, 95\%I 1.176-10.075; $P=0.024)$ as independent risk factors for PEP. Also, multivariate regression analysis failed to illustrate that intramuscular diclofenac prevented PEP (odds ratio 0.79; $95 \%$ confidence interval $0.39-1.25 ; P=0.51$ ).

The authors also did not encounter any adverse effect related to the study drug. Bleeding after endoscopic sphincterotomy was observed in $8.4 \%$ patients [12 $(9.4 \%)$ in the placebo 
group and $9(7.3 \%)$ in the diclofenac group $(P=0.507)]$ with all bleeding episodes being mild. There was one major complication (immediate duodenal perforation) in the diclofenac group that was treated with primary surgical closure. The authors concluded that prophylactic intramuscular diclofenac had no beneficial preventive effect on PEP.

\section{Commentary}

Prevention of PEP is one of the desired dreams of endoscopists and over last three decades a number of endoscopic procedures as well as drugs have been evaluated for prevention of PEP with varying success. Of all the available and tested drugs rectal indomethacin and diclofenac have been shown to reduce the severity of PEP and are recommended (as a grade A recommendation) by the European Society of Gastrointestinal Endoscopy for almost all patients undergoing ERCP. ${ }^{[10,11]}$ However, rectal preparations of NSAID are not easily available, are uncomfortable for the patients and are usually expensive. On the other hand, parenteral preparations of NSAID's are easily available and have a rapid onset of action as compared to other routes. Therefore, it is more tempting to use parenteral NSAID's for prevention of PEP but the available literature on this topic is scanty. One study showed benefit of intra muscular diclofenac in the patients without SOD. ${ }^{[9]}$ However, the current study has validated that that NSAIDs administered via non-rectal routes do not appear to be protective. ${ }^{[1]}$ Also, few previous studies using intravenous valdecoxib, and oral diclofenac have shown that these routes are not effective in preventing PEP. ${ }^{[12,13]}$ The efficacy of rectal NSAID's have been reinforced by a recent study that evaluated the risk of PEP in patients with failed pancreatic stent placement (FPS) and the impact of rectal indomethacin on this risk. ${ }^{[14]}$ The authors reported that the incidence of PEP among patients in the placebo group who experienced FPS was $34.7 \%$, significantly higher than the patients who underwent successful stent placement (16.4\%) and in those without a stent attempt (12.1\%). On the other hand in the rectal indomethacin group, only $1(5.3 \%)$ of the 19 patients with FPS developed PEP, compared with 23 of the 239 patients who underwent successful stent placement $(9.6 \% ; P=1.0)$ and 6 of the 58 patients who did not undergo a stent attempt $(10.3 \% ; P=1.0)$. Therefore, based on the currently available data, only rectal indomethacin or diclofenac should be used for prevention of PEP.
Surinder S Rana

Department of Gastroenterology, Post Graduate Institute of Medical Education and Research, Sector 12,

Chandigarh - 160 012, India

Address for correspondence:

Dr. Surinder Singh Rana, Department of Gastroenterology, PGIMER,

Chandigarh - 160 012, India

E-mail: drsurinderrana@yahoo.co.in

\section{References}

1. Andriulli A, Loperfido S, Napolitano G, Niro G, Valvano MR, Spirito F, et al. Incidence rates of post-ERCP complications: A systematic survey of prospective studies. Am J Gastroenterol 2007;102:1781-8.

2. $\quad$ Ding X, Zhang F, Wang Y. Risk factors for post-ERCP pancreatitis: A systematic review and meta-analysis. Surgeon 2014;pii:S1479666X(14)00146-2.

3. Lee TH, Park DH. Endoscopic prevention of post-endoscopic retrograde cholangiopancreatography pancreatitis. World J Gastroenterol 2014;20:16582-95.

4. Thaker AM, Mosko JD, Berzin TM. Post-endoscopic retrograde cholangiopancreatography pancreatitis. Gastroenterol Rep (Oxf) 2014. [In Print].

5. Wong LL, Tsai HH. Prevention of post-ERCP pancreatitis. World J Gastrointest Pathophysiol 2014;5:1-10.

6. Yuhara H, Ogawa M, Kawaguchi Y, Igarashi M, Shimosegawa T, Mine T. Pharmacologic prophylaxis of post-endoscopic retrogradecholangiopancreatography pancreatitis: Protease inhibitors and NSAIDs in a meta-analysis. J Gastroenterol 2014;49:388-99.

7. Elmunzer BJ, Waljee AK, Elta GH, Taylor JR, Fehmi SM, Higgins PD. A meta-analysis of rectal NSAIDs in the prevention of post-ERCP pancreatitis. Gut 2008;57:1262-7.

8. Elmunzer BJ, Scheiman JM, Lehman GA, Chak A, Mosler P, Higgins PD, et al. A randomized trial ofrectal indomethacin to prevent post-ERCP pancreatitis. N Engl J Med 2012;366:1414-22.

9. Senol A, Saritas U, Demirkan H. Efficacy of intramuscular diclofenac and fl uid replacement in prevention of post-ERCP pancreatitis. World J Gastroenterol 2009;15:3999-4004.

10. Dumonceau JM, Andriulli A, Elmunzer BJ, Mariani A, Meister T, Deviere J, et al. Prophylaxis of post-ERCP pancreatitis: European Society of Gastrointestinal Endoscopy (ESGE) guideline - Updated June 2014. Endoscopy 2014;46:799-815.

11. Elmunzer BJ. Nonsteroidal anti-infl ammatory drugs for preventing post-ERCP pancreatitis: Why rectal and not intramuscular? Endoscopy 2015;47:6-7.

12. Bhatia V, Ahuja V, Acharya SK, Garg PK. A randomized controlled trial of valdecoxib and glyceryl trinitrate for the prevention of post-ERCP pancreatitis. J Clin Gastroenterol 2011;45:170-6.

13. Cheon YK, Cho KB, Watkins JL, McHenry L, Fogel EL, Sherman S, et al. Efficacy of diclofenac in the prevention of post-ERCP pancreatitis in predominantly high-risk patients: A randomized double-blind prospective trial. Gastrointest Endosc 2007;66:1126-32.

14. Choksi NS, Fogel EL, Cote GA, Romagnuolo J, Elta GH, Scheiman JM, et al. United States Cooperative for Outcomes Research in Endoscopy. The risk of post-ERCP pancreatitis and the protective effect of rectal indomethacin in cases of attempted but unsuccessful prophylactic pancreatic stent placement. Gastrointest Endosc 2015;81:150-5. 